Proc: RHIC Physics and Beyond: Kay Kay Gee Day

October 23, 1998

BNL, Upton, NY

\title{
Parton Structure through Two Particle Correlations in Au-Au at RHIC
}

\author{
Ronald S. Longacre \\ Physics Department, Brookhaven National Laboratory, Upton, NY 11973, USA
}

\begin{abstract}
A method for determining the presence of partonic scattering through two-particle correlations is developed and applied to models which have jets and mini-jets in them. We only consider the correlation of mid-rapidity particles because they will be easily measured in large numbers at RHIC. The level of two-particle correlations will be a direct measure of how dense a system is made in Au-Au collisions at RHIC. The STAR TPC will be ideal for making these measurements in the first year of running.
\end{abstract}

\section{INTRODUCTION}

Collisions of high energy protons are dominated by $\mathrm{pQCD}$ (perturbative Quantum Chromo-Dynamics), where the parton picture of quarks and gluons is the main player with hadrons being the final state manifestation of the former. The well established relationship between partons and the fragmentation into hadrons (jets) is the underlyirg process that inust certainly take place in a ultra high energy $A A$ (nucleus nucleus) collision when one considers hadrons with large transverse momentum. In proton colliders the large transverse momentum of hadrons coming from jets are translated into transverse energy spikes in a cross sectional region of a energy measuring device (calorimeter), this cross sectional region is called a jet cone [1]. Thus in $p p$ collisions calorimeters can be used to measure jet cross sections which can be compared with predictions of pQCD [2]. The situation for jets in $A A$ collisions is alot harder because of the large number of soft particles which can contribute to transverse energy. In the jet cone region for central $\mathrm{Au}-\mathrm{Au}$ collisions at the RHIC collider $(39.4 \mathrm{TeV})$ the transverse energy is around $200 \mathrm{GeV}$. Even though this is only $1 / 2 \%$ of the total energy, it makes it very

1) This research was supported by the U.S. Department of Energy under Contract No. DE-AC02-98CH10886. 


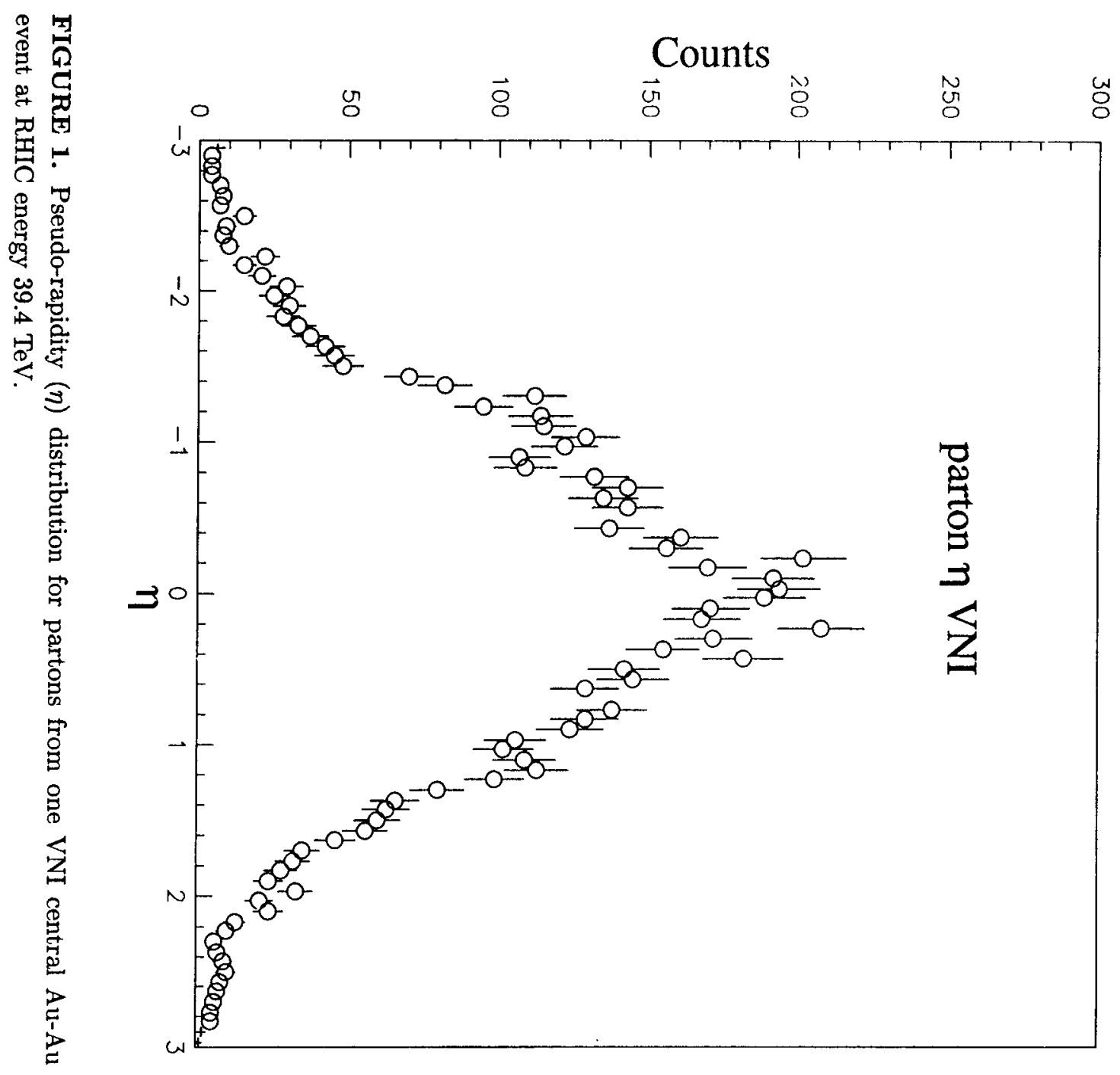




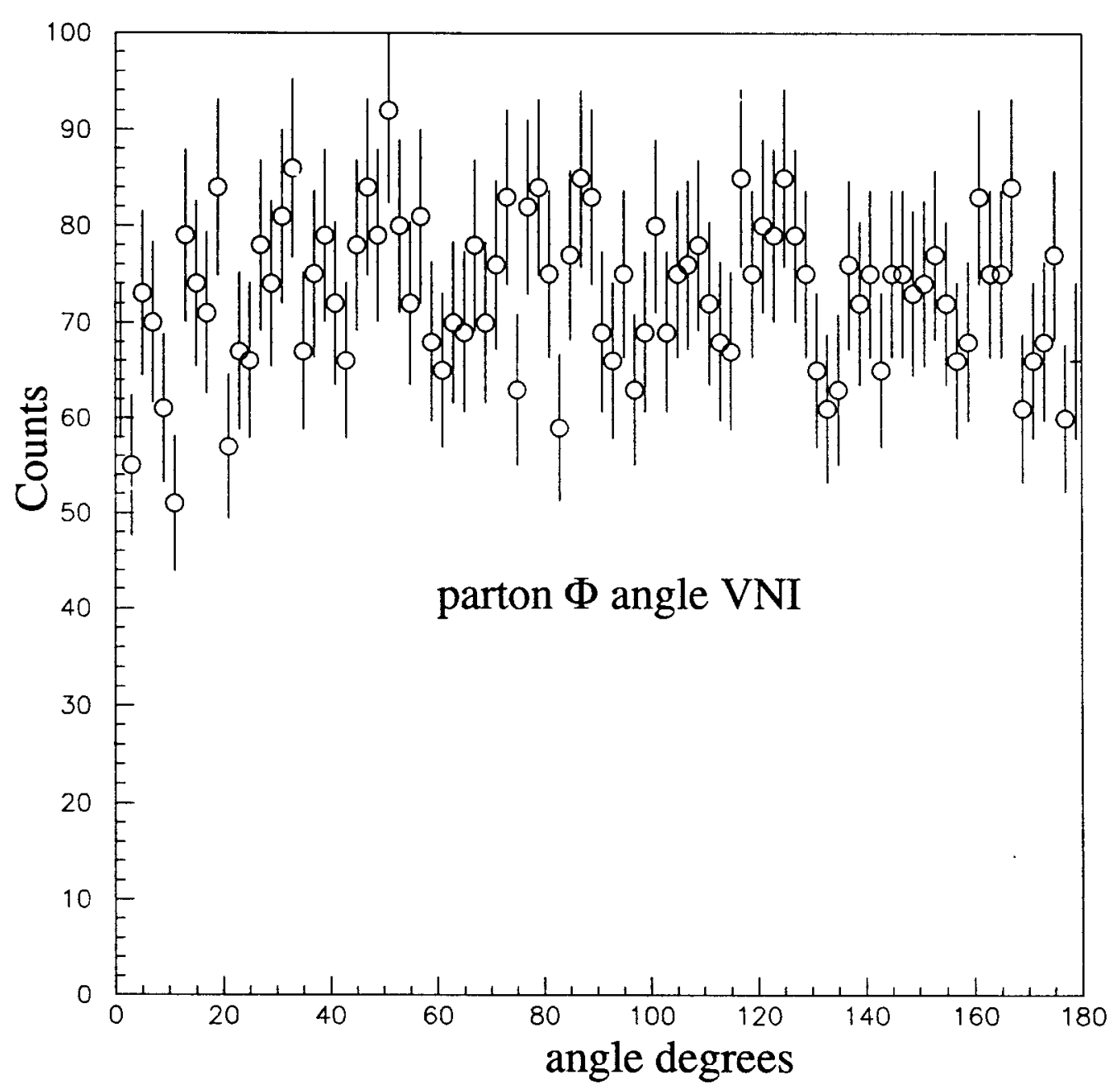

FIGURE 2. The $\phi$ angular distribution about the colliding Au-Au beams for one VNI central event at RHIC energy $39.4 \mathrm{TeV}$. 


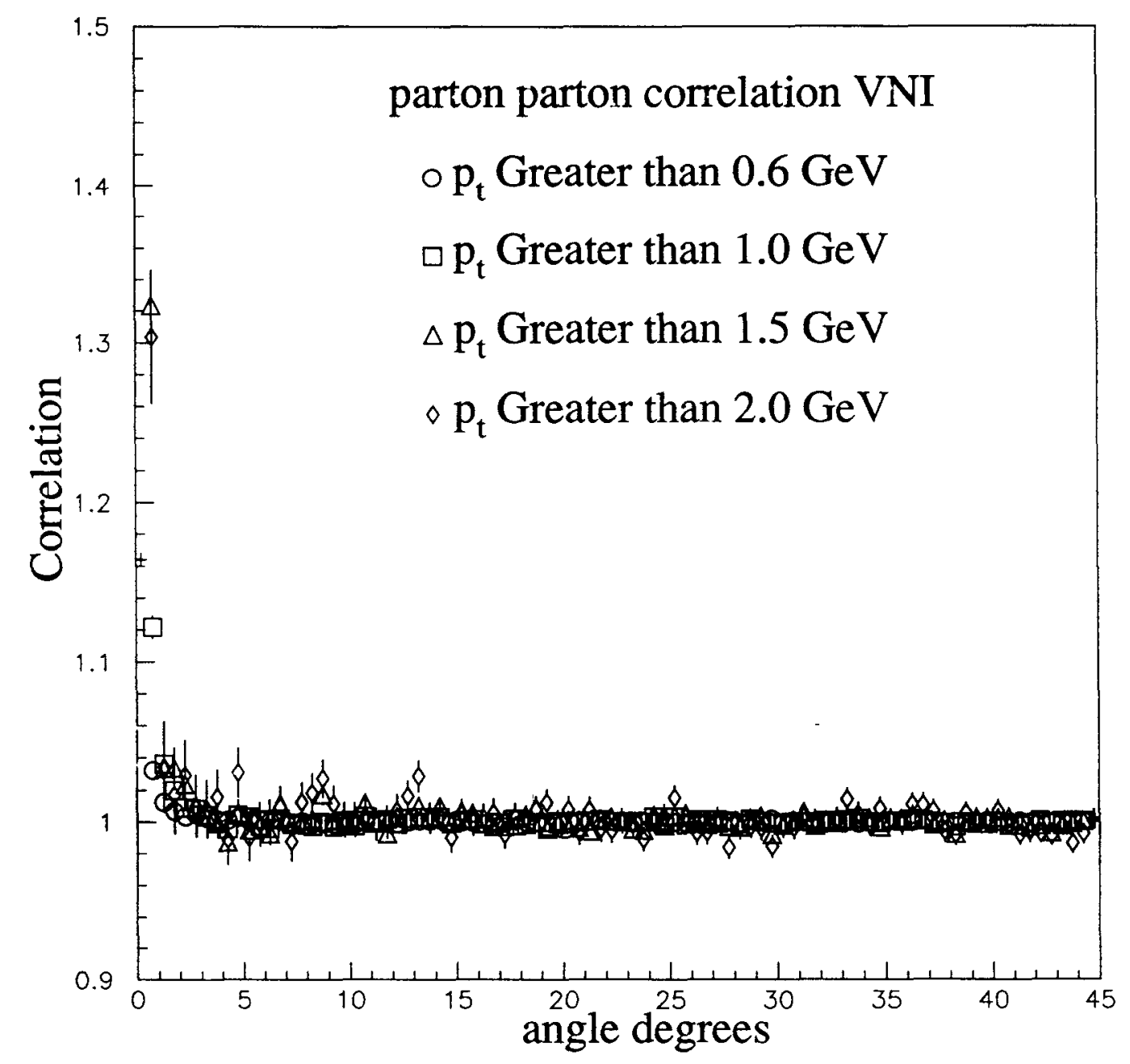

FIGURE 4. The parton parton angular correlation function for VNI central Au-Au RHIC events constructed from partons which have a $p_{t}$ : above $0.6 \mathrm{GeV} / \mathrm{c}$ (circles); above 1.0 $\mathrm{GeV} / \mathrm{c}$ (squares); above $1.5 \mathrm{GeV} / \mathrm{c}$ (triangles); and above $2.0 \mathrm{GeV} / \mathrm{c}$ (diamonds). 


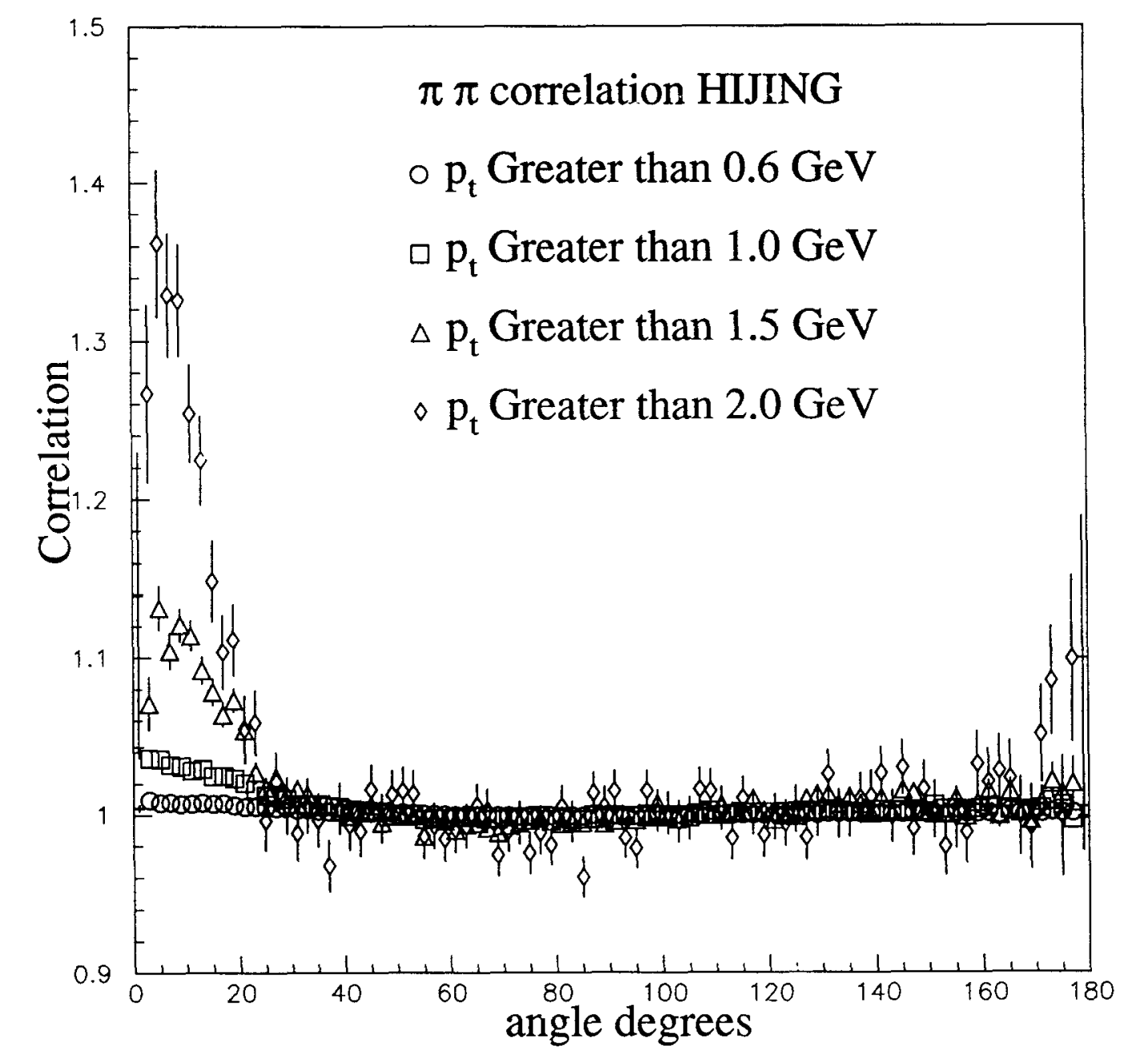

FIGURE 6. The $\pi \pi$ angular correlation function for HIJING central Au-Au RHIC events constructed from pions which have a $p_{t}$ : above $0.6 \mathrm{GeV} / \mathrm{c}$ (circles); above $1.0 \mathrm{GeV} / \mathrm{c}$ (squares); above $1.5 \mathrm{GeV} / \mathrm{c}$ (triangles); and above $2.0 \mathrm{GeV} / \mathrm{c}$ (diamonds). 


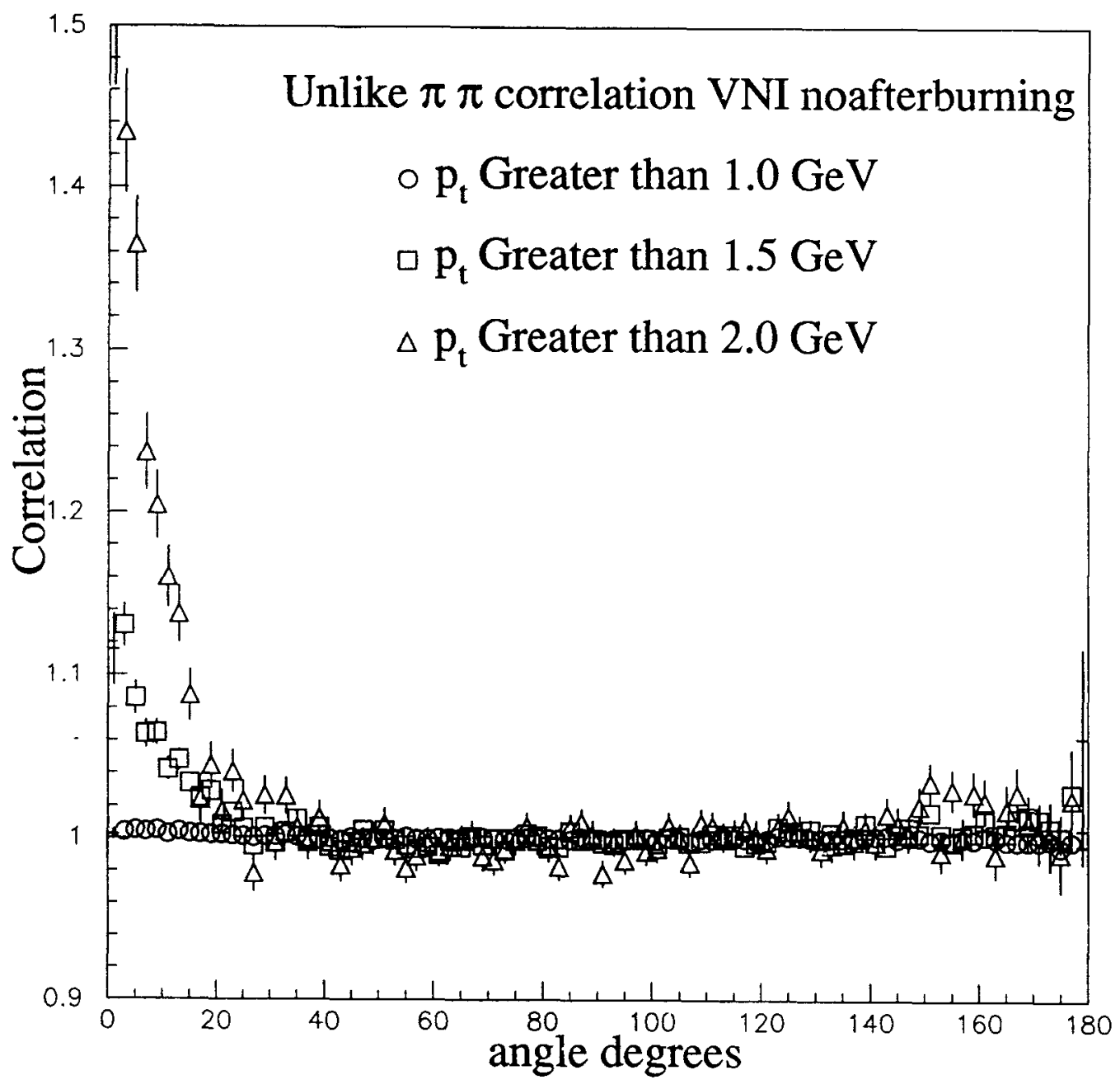

FIGURE 7. The unlike $\pi \pi$ angular correlation function for VNI central Au-Au RHIC events which do not have hadron rescattering constructed from pions which have a $p_{t}$ : above $1.0 \mathrm{GeV} / \mathrm{c}$ (circles); above $1.5 \mathrm{GeV} / \mathrm{c}$ (squares); and above $2.0 \mathrm{GeV} / \mathrm{c}$ (triangles). 


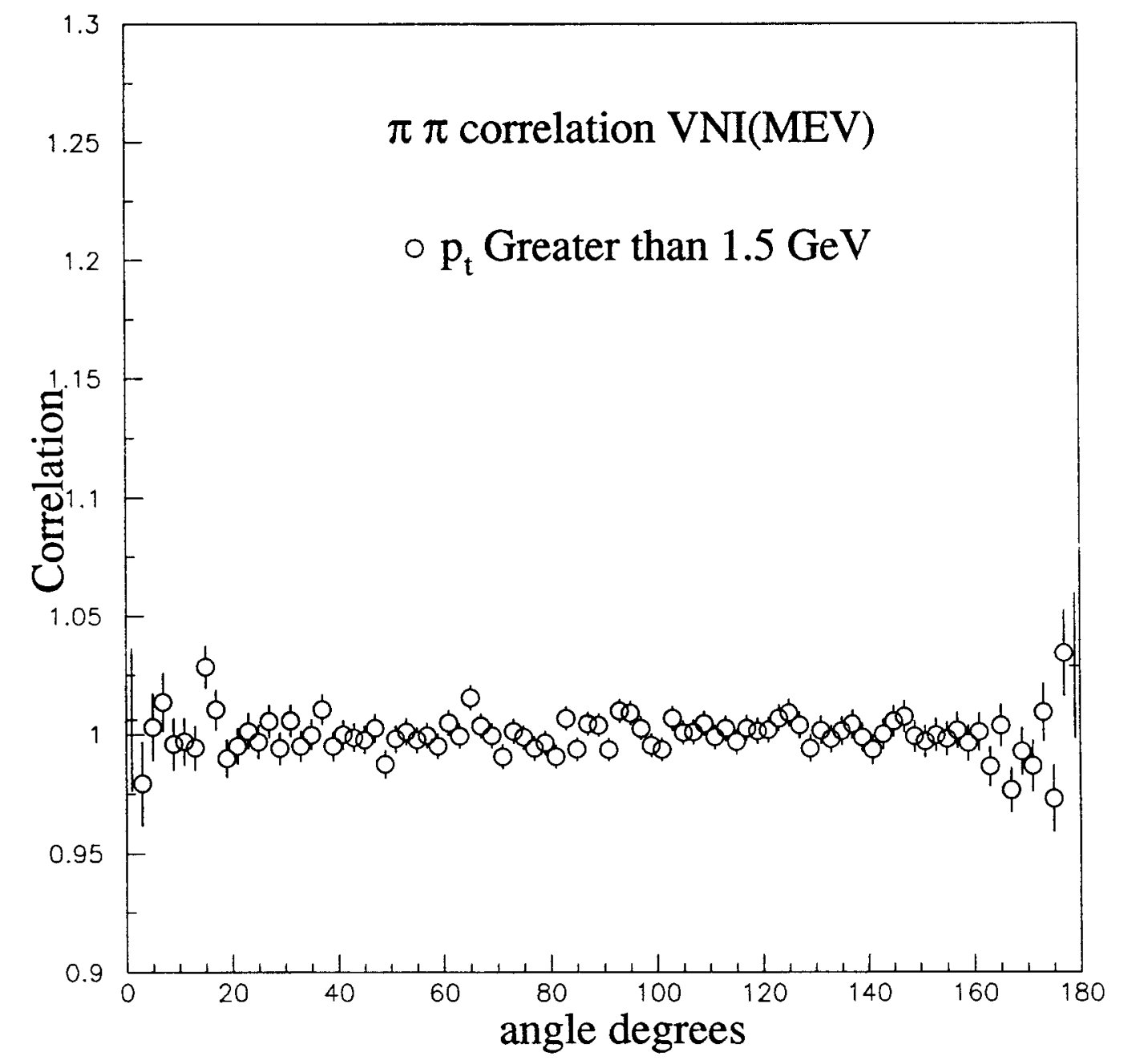

FIGURE 9. The $\pi \pi$ angular correlation function for MEV which is a program that simulates VNI central Au-Au RHIC events but does not have mini-jets constructed from pions which have a $p_{t}$ above $1.5 \mathrm{GeV} / \mathrm{c}$ (circles). 
(Fig. 14). In the STAR TPC the charged particle correlation will be very easily measured when one gets around 10,000 central Au-Au events. The comparison to Figs. 13 and 14 will show if the anticipated final state effects on mini-jets are greater or less than the to models give. One should go further and study the correlation as a function impact parameter. For the more peripheral collisions the mini-jet correlation should approach that of $p p$ collisions, since no final state in teractions can take place. As one reduces the impact parameter one should see a greater or less reduction of the correlation compared to the predictions of the models.

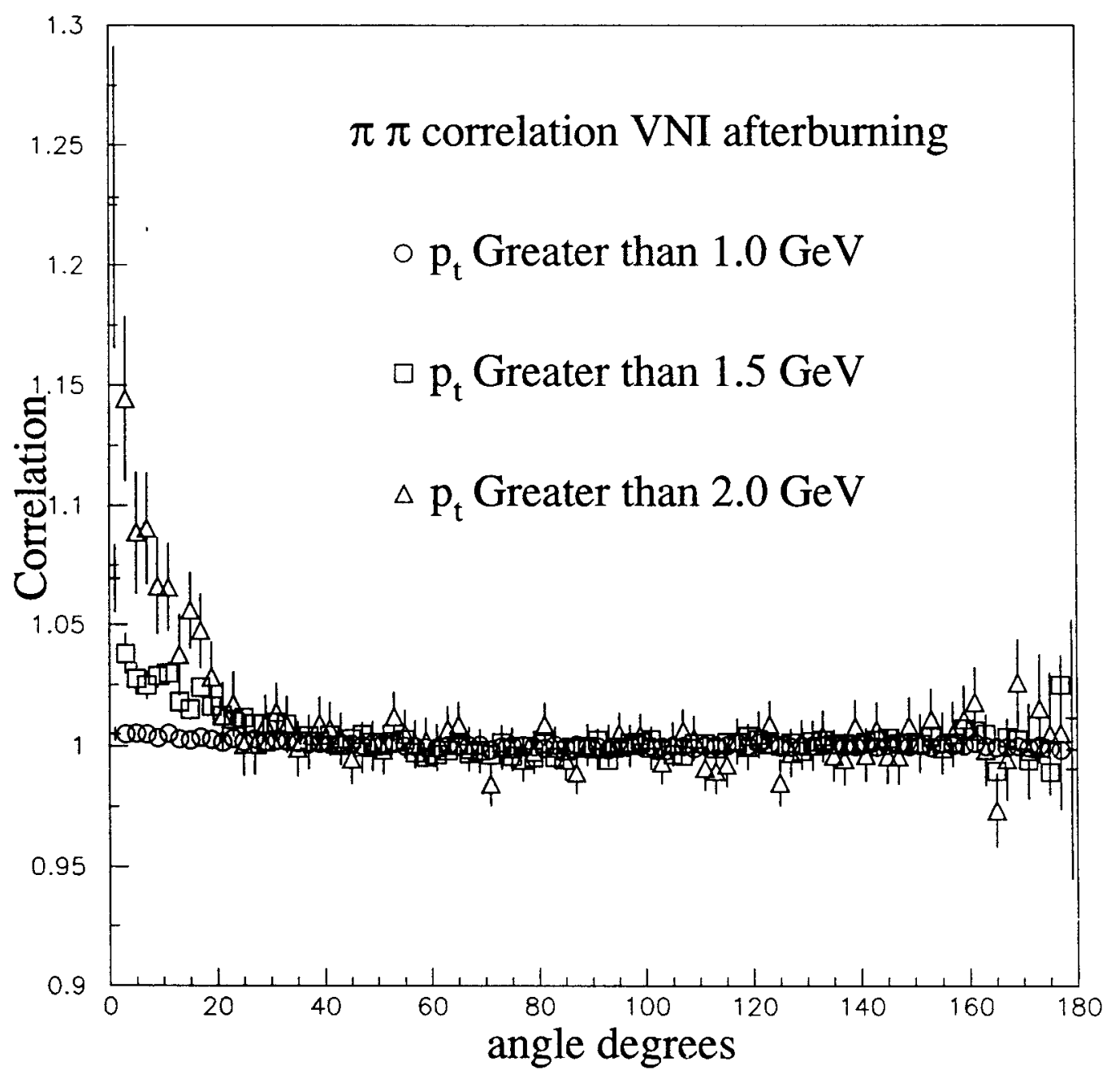

FIGURE 11. The $\pi \pi$ angular correlation function for VNI central Au-Au RHIC events which do have hadron rescattering constructed from pions which hove a $p_{t}$ : above $1.0 \mathrm{GeV} / \mathrm{c}$ (circles); above $1.5 \mathrm{GeV} / \mathrm{c}$ (squares); and above $2.0 \mathrm{GeV} / \mathrm{c}$ (triangles). 


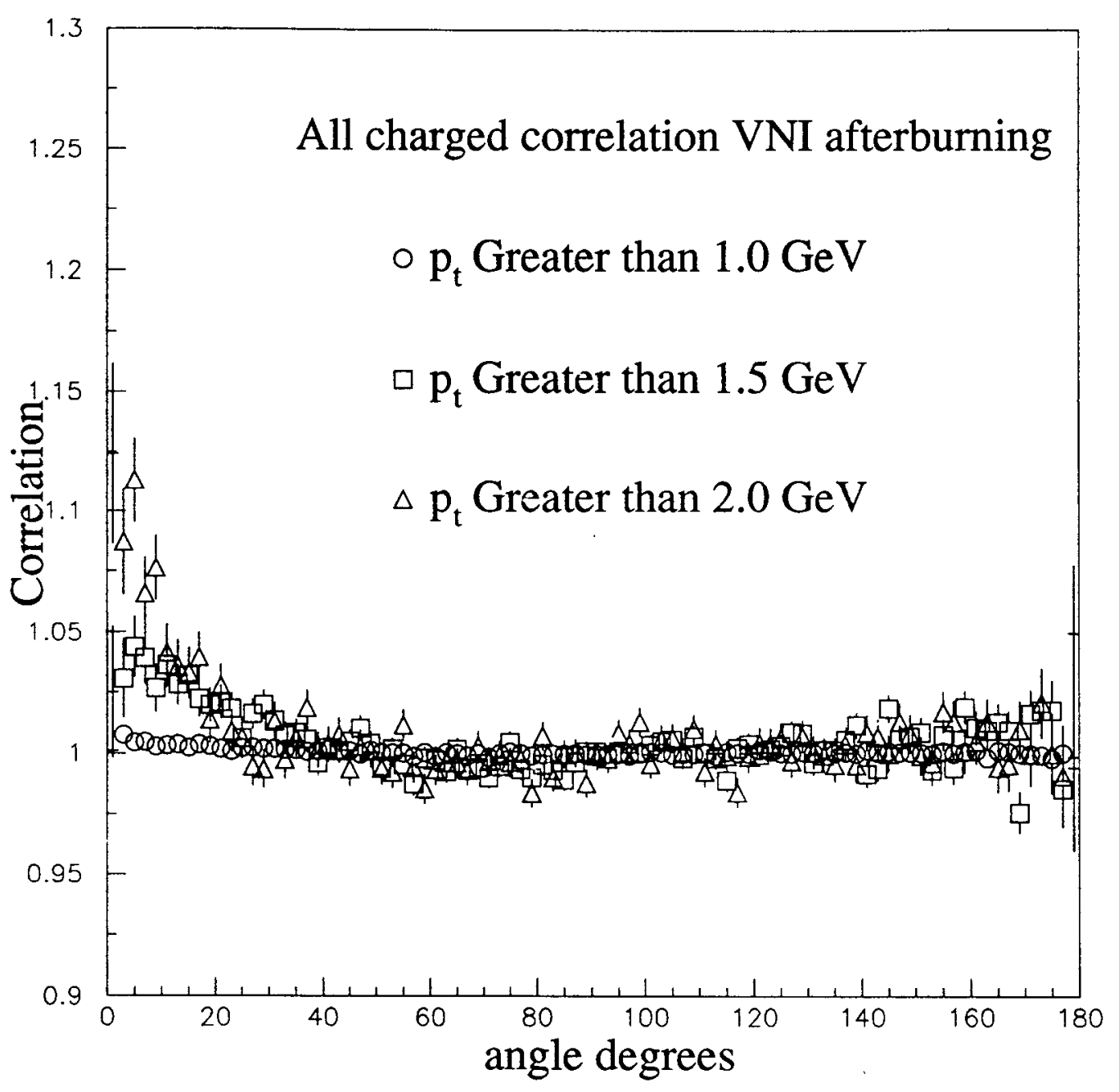

FIGURE 13. The charged particle angular correlation function for VNI central $\mathrm{Au}-\mathrm{Au}$ RHIC events which do have hadron rescattering constructed from partcles which have a $p_{t}$ : above $1.0 \mathrm{GeV} / \mathrm{c}$ (circles); above $1.5 \mathrm{GeV} / \mathrm{c}$ (squares); and above $2.0 \mathrm{GeV} / \mathrm{c}$ (triangles). 
collisions at RHIC. One will have to study $p p, p A$, and lighter $A A$ systems in order to understand how this type of correlation changes under different conditions. In the lighter systems one will have a direct measurement of jets to calibrate models and the correlation itself.

\section{REFERENCES}

1. S.Ellis, Z.Kunszt, and D.Soper, Phys. Rev. Lett. 62, 2188 (1989), Phys. Rev. Lett. 64, 2121 (1990).

2. G.Arnison et al., Phys. Lett. 132B, 214 (1983).

3. Klaus Geiger, Ron Longacre, and Dinesh K. Srivastava, VNI; Simulation of high-energy particle collisions in QCD, BNL-65755 (1998).

4. Klaus Geiger and Ron Longacre, Heavy Ion Phys. 8, 41 (1998).

5. X.N.Wang and M.Gyulassy, Phys. Rev. D44, 3501 (1991).

6. Lanny Ray (private communication). 